\title{
Helioseismic inferences on subsurface solar convection
}

\author{
Alexander G. Kosovichev \\ Stanford University, Stanford, CA 94305, USA
}

\begin{abstract}
Helioseismology has provided robust estimates of global properties of the solar convection zone, its depth, stratification, and revealed rotational shear layers at the boundaries. New methods of local helioseismology provide 3D maps of subsurface convective flows. In the quiet Sun regions, these maps reveal that supergranular-scale convection extends to the depth of 12-15 Mm. Analysis of evolution of the supergranular convection pattern shows evidence for a wave-like behavior which might be related to the interaction between convection and the subsurface rotational sheer layer. Helioseismology also reveals large-scale circulation flows around magnetic regions. These flows affect the evolution of the mean meridional flow during the solar cycle and, probably, the magnetic flux transport from mid-latitudes to the polar regions, a process important for solar dynamo theories. Helioseismic measurements on a smaller scale, below sunspots, give insight on how convection interacts with strong magnetic fields.
\end{abstract}

Keywords. Sun: helioseismology, convection, Sun: rotation

\section{Introduction}

Solar oscillations excited by turbulent convection below the solar surface provide unique information about properties of solar convection. Helioseismology provided first measurements of the depth of the convection zone in 1991 obtained by Christensen-Dalsgaard et al. (1991), Kosovichev \& Fedorova (1991), and during the past 15 years sophisticated tools were developed to measure accurately various properties of solar convection including the structure and dynamics at the bottom and top boundaries (the so-called tachocline and the subsurface shear layer), supergranulation, large-scale convective flows, magnetoconvection in sunspots and flow patterns around active regions. The helioseismology results provided first insight into the physical properties of solar convection and its role in the Sun's magnetic activity. They challenge the current theories of solar convection and stimulate further observational and theoretical studies. In this article, I present a brief summary of some major helioseismology results.

\section{Global and local helioseismology}

Two different approaches in helioseismology have been developed. These are broadly called "global" and "local" helioseismology. Global helioseismology is based on inversion of frequencies of normal oscillation modes. The oscillation power spectrum of the Sun (Fig. 1) includes surface gravity (f) and acoustic (p) modes, observed in the range of angular degree $\ell$ from 0 to about 3000. In the low- and medium-degree range, $\ell=0-300$, the oscillation frequencies are measured to a very high precision $\left(\sim 10^{-5}-10^{-6}\right)$, and used for accurate inversions of the solar structure and rotation. The high-frequency modes are much more difficult to measure and interpret because the peaks of neighboring modes are merged together in the power spectrum, and also because these modes strongly interact with near surface turbulence and the atmosphere. 

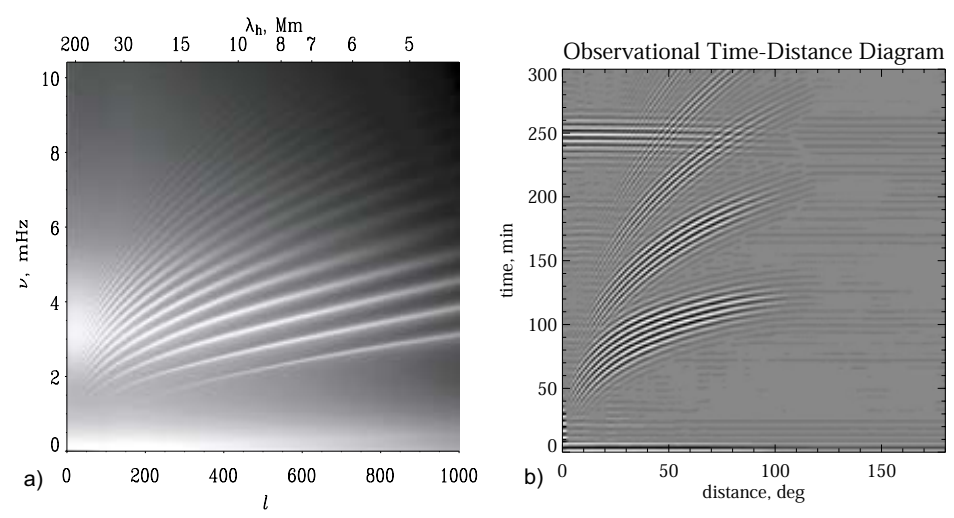

Figure 1. (a) The power spectrum of solar oscillations as a function of angular degree $\ell$ (or horizontal wavelength $\lambda_{h}$ ), cyclic frequency $\nu$; the lowest ridge corresponds to the surface gravity (f) mode, the upper ridges are acoustic (p) modes; $(b)$ the time-distance cross-covariance function as a function of the horizontal travel distance and the lag time; the lowest ridge corresponds to the direct waves traveling between two surface points through the interior; the upper ridges correspond to waves with multiple bounces.

Local helioseismology is based on measurements of local properties of solar oscillations such as frequency shifts in the power spectra calculated for small areas and travel times of solar waves This method is called ring-diagram analysis, e.g. Gough \& Toomre (1983), Haber et al. (2002). Despite the stochastic nature of solar oscillations it is possible to measure travel times for packets of $\mathrm{p}$ - and f-modes by calculating a cross-covariance function of the oscillation signals observed at different points on the Sun, for sufficiently long time, typically 8 hours. The cross-covariance plotted as a function of the distance between the points and the time lag displays a set of ridges which correspond to wave packets propagating between these points through the interior. The lowest ridge corresponds to direct waves propagating between two surface points through the interior. The wave penetration depth is approximately $1 / 3$ of the travel distance on the surface. The measured travel times are used to infer the sound speed variations and velocity of mass flows in the region of wave propagation. This technique, called time-distance helioseismology, is being actively developed and used for measuring local properties of solar convection in the upper convection zone, e.g. see Duvall et al. (1993), Kosovichev (1996a), Kosovichev \& Duvall (1997), Gizon et al. (2001), Zhao \& Kosovichev (2004). Diagnostics of deep convection with this and also other local helioseismology techniques have not been developed.

\section{Inferences from global helioseismology}

\subsection{Depth of the convection zone}

The depth of the solar convection zone is defined as a transition point from the almost adiabatic stratification of the convection zone to the subadiabatic radiative interior. In standard solar models at this point the first derivative of the sound speed $(c)$ changes its slope, and the parameter $W=\left(r^{2} / G M_{\odot}\right)\left(d c^{2} / d r\right)$, which is constant in the convection zone $(\approx 2 / 5)$ has a discontinuous first derivative (Fig. 2$)$. This property of the soundspeed was used to define the depth of the convection zone by Christensen-Dalsgaard et al. (1991), who determined that the depth is $\approx 0.287 \pm 0.003 R_{\odot}$ by using asymptotic inversions for the sound speed profile (Fig. $2 \mathrm{~b}$ ). This value was confirmed by Kosovichev \& Fedorova (1991)(Fig. 2a). 

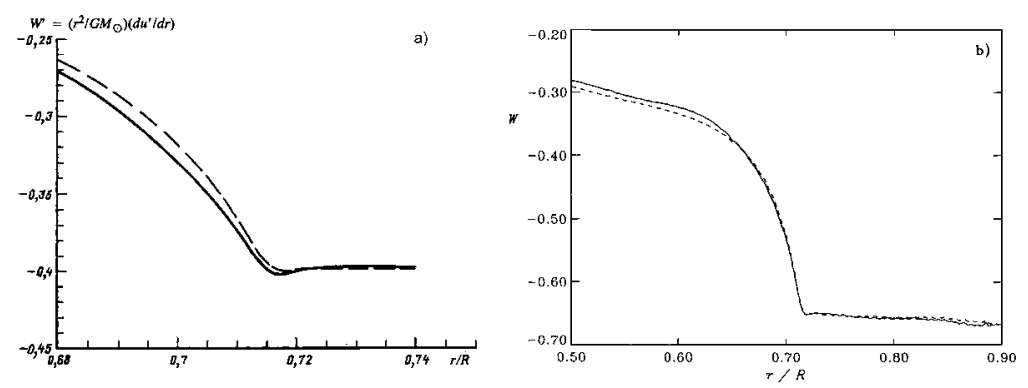

Figure 2. The parameter $W=\left(r^{2} / G M_{\odot}\right)\left(d c^{2} / d r\right)$, which defines the depth of the convection zone, as a function of radius determined by helioseismic inversions for the Sun (solid curves) and for a reference solar model (dashed curves) by (a) Kosovichev \& Fedorova (1991) and (b) Christensen-Dalsgaard et al. (1991).

The convection zone may be slightly prolate (Gough \& Kosovichev 1995).

\subsection{Convective overshoot and tachocline}

Convection theories predict convective overshoot regions at the convection zone boundaries. As a result of the overshoot at the bottom boundary the region of constant $W$ could extend deeper into the radiative zone leading to a discontinuity of $W$. However, such zone of adiabatic overshoot was not found. The upper limit for this is $\approx 2.8 \mathrm{Mm}$ $\left(0.004 R_{\odot}\right)$ (Basu 1997). Another possibility is that the mean structure of the overshoot zone remans subadiabatic, where the sound speed is only slightly increased. This may happen, for instance, if overshoot consists of sparsely distributed plumes. It is quite possible that the sharp peak in the deviation of the Sun's sound speed from the standard model is caused by such subadiabatic overshoot (Fig. 3).

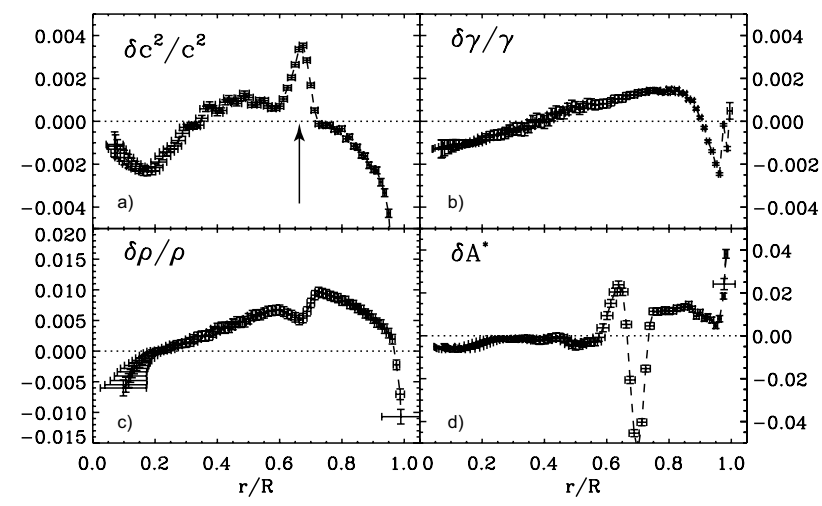

Figure 3. The relative difference between the inferred solar properties and the standard solar model S of Christensen-Dalsgaard et al. (1996) for: (a) the squared sound speed; $(b)$ the adiabatic exponent; $(c)$ the mass density; and $(d)$ the parameter of convective stability $A^{*}=\frac{1}{\gamma} \frac{d \log p}{d \log r}-\frac{d \log \rho}{d \log r}$, from Kosovichev (1999).

Splitting of the mode frequencies, which is sensitive to latitudinal variations, shows that the sound speed enhancement is essentially spherically symmetrical. Thus, it is less likely to be caused by instabilities of the differential rotation. The thickness of this layer is about $0.019 R_{\odot}$ (Elliott \& Gough 1999).

The zone of subadiabatic overshoot coincides with a sharp change of the solar rotation law, from the differential rotation in the convection zone to almost solid-body rotation 
in the radiative interior (Fig. 4). This so-called tachocline is located mostly below the adiabatic boundary of the convection zone. The tachocline may play a key role in the generation of magnetic fields on the Sun. Its dynamics is discussed in the review by Christensen-Dalsgaard in these Proceedings.
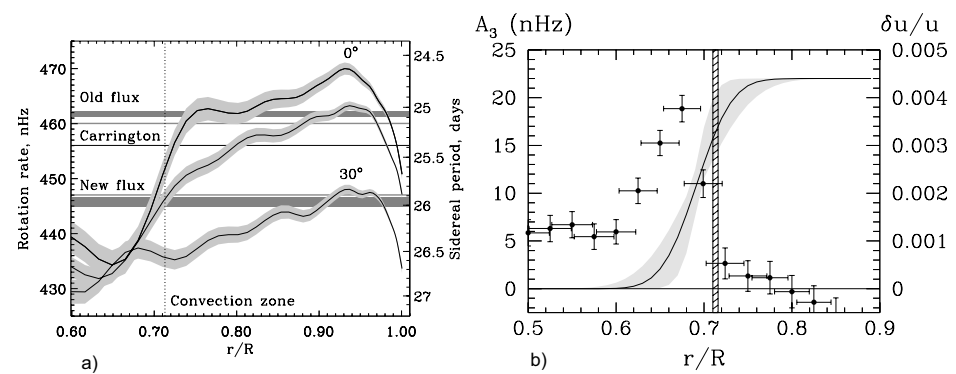

Figure 4. a) The rotation rate inside the Sun as a function of radius at three latitudes, 0,15 and 30 degrees; vertical line indicates the bottom of the convection zone; horizontal lines shows the rotation rate of magnetic fluxes and rotation rate of sunspots at $16^{\circ}$ (Carrington rotation), from Benevolenskaya et al. (1999); (b) $A_{3}$ (solid curve) parameter of the differential rotation law showing the tachocline; the crosses show the parameter $u=p / \rho$ (proportional to $c^{2}$ ); the vertical shaded bar shows the location of the bottom of the convection zone (Kosovichev 1996b).

\subsection{Sub-surface shear layer and seismic radius}

The helioseismology inversion results (Schou et al. 1997) shown in Fig. 4a also reveal a sharp decrease of the rotation rate just below the surface. This subsurface shear layer probably also plays an important role in the solar dynamics and magnetism. Its origin is not understood, but it was suggested that it may be related to the angular momentum transport by the near-surface convective flows. The thermodynamic structure of this layer has not been determined with sufficient precision because of the difficulties with measuring oscillation modes of high degree. In particular, the structure and extent of the superadiabatic layer of solar convection have not been measured.

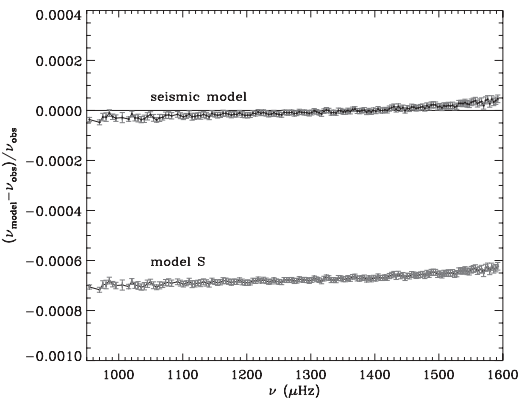

a)

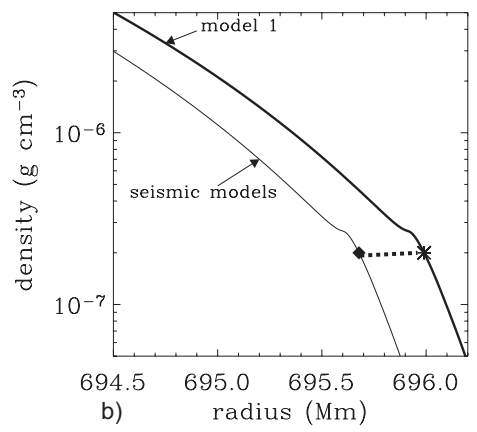

b) radius $(\mathrm{Mm})$

Figure 5. (a) The relative difference between the model and observed $f$-mode frequencies (lower curve with errorbars), and this difference for a model calibrated to the "seismic" solar radius; (b) the density profiles in the standard solar model (model 1) calibrated to the photospheric radius, and in the seismic model calibrated to the seismic radius; the dashed line indicates a possible extension of the superadiabatic convection layer to satisfy both the helioseismic and surface data (Schou et al. 1998).

It is interesting to note that observations of the $f$-mode show that the frequencies of this mode are systematically higher than the frequencies calculated for a standard solar model (Fig. 5a). This mode is essentially insensitive to thermodynamic properties, and its 




Figure 6. A map of horizontal flow velocities in the upper convection zone (depth 1-2 Mm); the dark and bright spots show the magnetic regions of north and south polarities; the longest arrow corresponds to $\approx 1 \mathrm{~km} / \mathrm{s}$. From Zhao \& Kosovichev (2004).


Figure 7. Wave-like behavior of supergranulation: $(a)$ the observed dispersion $(\ell-\nu)$ relation for supergranulation of Gizon et al. (2003); (b) a schematic picture of convection in a stratified shear layer by Adam (1977); (c) results of calculations of the phase speed of convective modes as a function of the velocity gradient in the shear layer (Green \& Kosovichev 2006).

frequency depends mostly on the solar radius determined by the location of the density gradient just below the surface (Fig. 5b). Calibration of the solar models to the observed $f$-mode frequencies results in a reduction in the surface radius by $\sim 300 \mathrm{~km}$ relative to the standard photospheric radius determined from limb observations (Schou et al. 1998). If this radius is correct then we have to assume that the superadiabatic zone is wider than according to the mixing length theory, perhaps, because of overshooting at the top. A possible extension of this zone is illustrated by the dashed line in Fig. 5b.

\section{Inferences from local helioseismology}

\subsection{Supergranulation}

Supergranulation plays a very important role in solar dynamics because it effectively transports magnetic flux and maintains the chromospheric network. Time-distance 


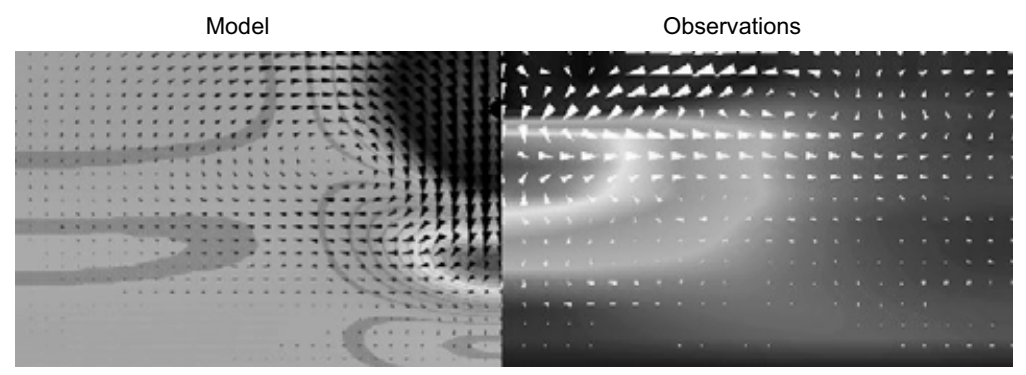

a)

b)

Figure 8. Comparison of the subsurface structure and flow patterns below sunspots, obtained from (a) an MHD model of Hurlburt \& Rucklidge (2000) and (b) time-distance helioseismology results of Kosovichev et al. (2000), Zhao et al. (2001). The grayscale images show the distribution of temperature for the model and the sound speed for the observations; the arrows show the flow velocities; the largest arrow in the observations corresponds to $\approx 2 \mathrm{~km} / \mathrm{s}$.

helioseismology has revealed the structure of supergranulation below the surface (Fig. 6) (Kosovichev \& Duvall 1997; Duvall et al. 1997; Zhao \& Kosovichev 2004). It has been determined that the diverging horizontal supergranulation flows extend into the deeper layers, and that the return converging flows are formed at a depth of 12-16 Mm. However, the structure of the vertical flows has not been measured because these are much weaker than the horizontal flows, and the measurements are affected by a cross-talk effect.

A puzzling property is that the supergranulation pattern rotates faster than the surface plasma and faster than all magnetic structures, and even shows a wave-like behavior with a specific dispersion relation (Fig. 7a) (Gizon et al. 2003). A possible explanation is that the wave-like behavior is caused by the subsurface shear flow (Fig. 7b). Calculations in the linear approximation of Green \& Kosovichev (2006) show that such effects exist, but the phase speed of the traveling convective cells is lower than the observed phase speed $(25 \mathrm{~m} / \mathrm{s}$ vs. $65 \mathrm{~m} / \mathrm{s})$. Thus, non-linear modeling is necessary to study this property.

\subsection{Magnetoconvection in sunspots}

Local helioseismology measurements of the subsurface structure and flows in sunspot regions are somewhat uncertain because of the wave interaction with strong magnetic fields. Nevertheless, the initial results reveal complicated structures and flow patterns. While the $f$-mode measurements show an outflow generally consistent with the surface Evershed effect and the moat flow (Gizon et al. 2001), the results from the $p$-modes indicate that there are subsurface vortexes converging around sunspots, in the upper layer 4-5 Mm deep (Fig. 8b). The flow velocity reaches $2 \mathrm{~km} / \mathrm{s}$. In that layer the sound speed is lower than in the quiet Sun. In the deeper interior, the sound speed becomes higher, and the flow pattern is reversed. This picture is qualitatively consistent with the numerical MHD model of Hurlburt \& Rucklidge (2000).

\subsection{Large-scale flows around active regions}

On a the scale of active regions, local helioseismology results show a similar flow pattern: converging flows in the upper 0-5 Mm and diverging at depth 10-15 Mm. These flows are much weaker reaching only about $50 \mathrm{~m} / \mathrm{s}$. However, they may be strong enough to affect the global circulation of the Sun (Haber et al. 2004, Zhao \& Kosovichev 2004).

\subsection{Meridional circulation}

The synoptic maps of subsurface convective flows (such as shown in Fig. 6 and 9) can be used for deriving various global properties of the Sun's dynamics. Figure 10 shows the 

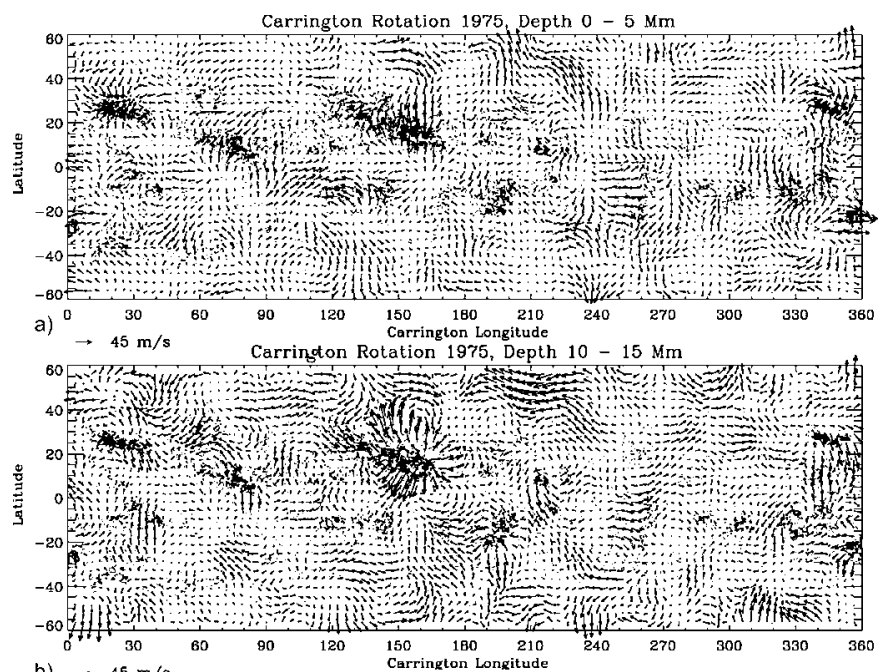

b) $\rightarrow 45 \mathrm{~m} / \mathrm{s}$

Figure 9. Synoptic flow maps showing large-scale flows around active regions: the flows converge in the upper layer 0-5 Mm deep (a); and diverge in the deeper layers, 10-15 Mm (b). The typical flow velocity is $50 \mathrm{~m} / \mathrm{s}$. From Zhao \& Kosovichev (2004).

differential rotation, meridional flow, vorticity, and zonal flows (calculated as a deviation from a smooth differential rotation law) as a function of sine latitude in the subsurface 1-2 Mm deep layer.
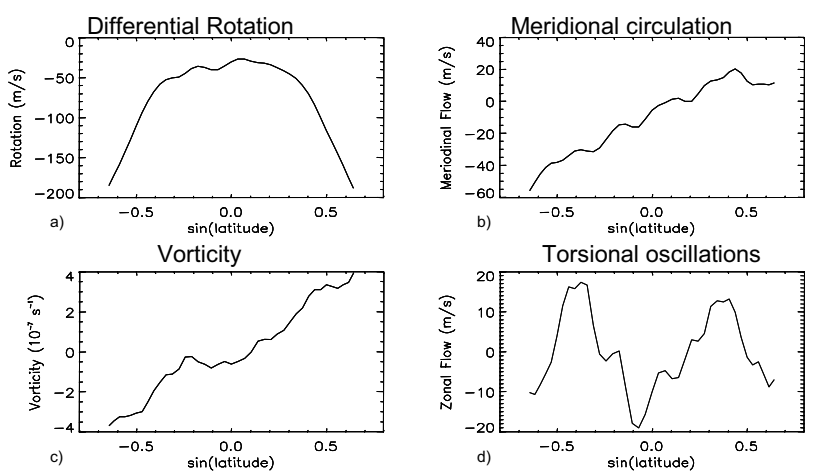

Figure 10. The azimuthally averaged properties of solar dynamics as a function of latitude derived from the subsurface synoptic flow maps (1-2 Mm deep): (a) the differential rotation profile; (b) meridional circulation; $(c)$ vorticity; (d) zonal flows ("torsional oscillations"), representing a deviation from a smooth rotation law. From Zhao \& Kosovichev (2004).

These properties are calculated by averaging the synoptic flow map. For understanding of the global dynamics of the solar convection zone it is important to investigate how these properties change during the solar cycle. It is particularly interesting that the meridional circulation changes quite significantly. The mean velocity of this flow decreases at mean and high latitudes during the solar maximum of 2000-2 (Fig. 11). This decrease is caused by the large-scale converging flows around active regions discussed in the previous section.

Thus, the helioseismology results reveal strong links between small, large and global scales of solar convection. 

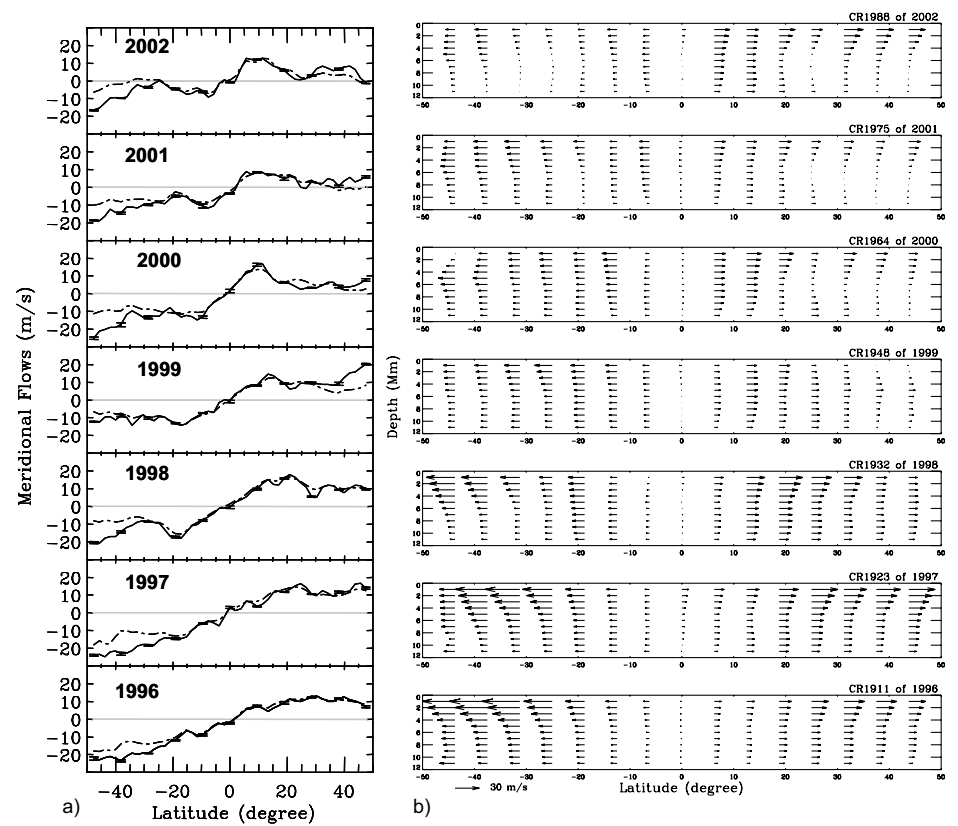

b)

Figure 11. Variation of the meridional flows during the solar cycle: $(a)$ flow velocity as a function of latitude at two depths, 3-4.5 Mm (solid curves) and 6-9 Mm (dashed curves); (b) flow velocity as a function of latitude and depth. From Zhao \& Kosovichev (2004).

\section{References}

Adam, J.A. 1977, Astrophys.Sp.Sci. 50, 493

Basu, S. 1997, MNRAS 288, 572

Benevolenskaya, E.E., et al. 1999, ApJL 517, L163

Christensen-Dalsgaard, J., Gough, D.O. \& Thompson, M.J. 1991, ApJ 378, 413

Christensen-Dalsgaard, J., et al. 1996, Science 272, 1286

Duvall, T.L., Jr., Jefferies, S.M., Harvey, J.W. \& Pomerantz, M.A. 1993, Nature 362, 430

Duvall, T.L., Jr., et al. 1997, Sol.Phys. 170, 63

Elliott, J.R. \& Gough, D. O. 1999, ApJ 516, 475

Gizon, L., Duvall, T.L., Jr. \& Larsen, R.M. 2001, IAU Symposium 203, 189

Gizon, L., Duvall, T.L. \& Schou, J. 2003, Nature 421, 43

Gough, D.O. \& Toomre, J. 1983, Sol.Phys. 82, 401

Gough, D.O. \& Kosovichev, A.G. 1995, in: Helioseismology ESA SP, p.47

Green, C.A. \& Kosovichev, A.G. 2006, ApJL 641, L77

Haber, D.A., et al. 2002, ApJ 570, 855

Haber, D.A., Hindman, B.W., Toomre, J. \& Thompson, M.J. 2004, Sol.Phys. 220, 371

Hurlburt, N.E. \& Rucklidge, A.M. 2000, MNRAS 314, 793

Kosovichev, A.G. \& Fedorova, A.V. 1991, Sov. Astron. 35, 507

Kosovichev, A.G. \& Duvall, T.L., Jr. 1997, ASSL Vol. 225: SCORe'96 : Solar Convection and Oscillations and their Relationship, 241

Kosovichev, A.G. 1996a, ApJL 469, L61

Kosovichev, A.G. 1996, ApJL 469, L61

Kosovichev, A.G. 1999, J. Comp. Appl. Math 109, 1

Kosovichev, A.G., Duvall, T.L., Jr. \& Scherrer, P.H. 2000, Sol.Phys. 192, 159

Schou, J., Kosovichev, A.G., Goode, P.R. \& Dziembowski, W.A. 1997, ApJL 489, L197

Schou, J., et al. 1998, ApJ 505, 390

Zhao, J., Kosovichev, A.G. \& Duvall, T.L., Jr. 2001, ApJ 557, 384

Zhao, J. \& Kosovichev, A.G. 2004, ApJ 603, 776 


\section{Discussion}

NANDI: The sound speed is expected to be modified when the waves interact with magnetic fields. Is this taken into account when inversions are done to infer local flows in sunspot structures?

Kosovichev: Yes, this is taken into account. The effects of the sound-speed perturbations and local flows are separated by measuring reciprocal travel times of acoustic waves traveling between two points in opposite directions. By taking the difference of these travel times we cancel the contribution of the sound-speed variations, and then infer the flow velocities by inversion of the travel-time differences.

RÜDIGER: The advection-dominated dynamo lives from the induction by the meridional flow at the bottom of the convection zone. Magnetically induced fluctuations of the surface flow do not strongly influence the dynamo process.

Kosovichev: Observations show that magnetic flux transport by meridional flows from the mid-latitude active regions to the polar regions of the Sun plays an important role in the solar dynamo and 11-year cycle. Properties of the near-surface meridional flow have been measured by the local helioseismology techniques. In particular, it has been established that the mean meridional flow changes during the solar cycle, mostly because of large-scale circulation cells developing around active regions during the activity maximum. These variations of the meridional flow (shown in Fig. 11) significantly affect the magnetic flux transport. The meridional flows at the bottom of the convection zone have not been measured. This task is planned for the Solar Dynamics Observatory mission.

TOOMRE: What are the prospects of probing the tachocline better as the Solar Dynamics Observatory and HMI data become available?

Kosovichev: The Helioseismic and Magnetic Imager (HMI) on SDO will observe the solar oscillations continuously with high spatial resolution. This will allow us to measure travel times of acoustic waves, which propagate to the bottom of the convection zone and cover large distances on the solar surface, more reliably, and thus significantly improve the helioseismic diagnostics of the tachocline. In particular, it will be important to detect longitudinal sound-speed variations related to non-axisymmetrical modes of the solar dynamo, and also measure the meridional flows in the tachocline.

Tikhomolov: Could you say, at least approximately, which one is deeper: overshoot or tachocline?

Kosovichev: I think that the tachocline essentially coincides with the overshoot zone. The location of the overshoot zone (which is subadiabatic) is determined by the sharp peak in the sound-speed profile at the bottom of the convection zone. This peak coincides with the sharp gradient of the differential rotation rate (the tachocline) as shown in Fig. 4b.

Martinez Pillet: Are sunspots disconnected from the tachocline as they get older?

Kosovichev: Unfortunately, we have not seen yet the deep roots of sunspots and do not know whether sunspots are connected to the tachocline or disconnected from it. We hope to solve this problem by using new helioseismology data from the Solar Dynamics Observatory. 